\title{
Matching, an Appealing Method to Avoid Confounding?
}

\author{
Michiel A. de Graaf ${ }^{a}$ Kitty J. Jager ${ }^{b}$ Carmine Zoccalic Friedo W. Dekker ${ }^{a}$ \\ a Department of Clinical Epidemiology, Leiden University Medical Centre, Leiden, bERA-EDTA Registry, \\ Department of Medical Informatics, Academic Medical Center, University of Amsterdam, Amsterdam, \\ The Netherlands; ${ }^{C}$ CNR-IBIM, Clinical Epidemiology and Pathophysiology of Renal Diseases and Hypertension, \\ Renal and Transplantation Unit, Ospedali Riuniti, Reggio Calabria, Italy
}

\section{Key Words}

Confounding $\cdot$ Matching $\cdot$ Individual matching $\cdot$ Frequency matching

\begin{abstract}
Matching is a technique used to avoid confounding in a study design. In a cohort study this is done by ensuring an equal distribution among exposed and unexposed of the variables believed to be confounding. In a matched casecontrol study, a case, affected by the disease, is matched with one or more individuals not affected by the disease, the controls. Because in a matched case-control study case and control group become too similar not only in the distribution of the confounder but also in the distribution of the exposure, one finds a lower effect estimate (odds ratio closer to 1). A matched case-control study requires statistical analysis to correct for this phenomenon. Nonetheless, a matched casecontrol study is suitable for confounders that are difficult to measure.

Copyright $\odot 2011$ S. Karger AG, Basel
\end{abstract}

A previous paper in this series discussed the problems of confounding. A confounder was described as a variable associated with both outcome and exposure without being an intermediate cause in the causal pathway between exposure and outcome. As described in that paper, a way to correct for a confounder is by removing its effect with statistical analysis, either by stratification or multivariate analysis [1]. A future paper will discuss these subjects. There are also ways to prevent confounding in the design of the study, namely randomization, restriction and matching. This paper will discuss the last option, matching.

\section{Basic Principles}

If we would perform a cohort study on the relation between hypertension and end-stage renal disease (ESRD), obesity is a potential confounder as obesity is associated with both hypertension and ESRD. As hypertension does not cause obesity, it is not in the causal pathway and hypertension indeed is a potential confounder [2]. If we would address this problem by matching, we would ensure that the average body mass index (BMI) is the same in the group of patients exposed to hypertension and another group of patients unexposed to hypertension. This could be achieved by searching an obese patient without hypertension for each obese patient with hypertension.

To get rid of this confounder in a cohort study a researcher can ensure an equal distribution among exposed and unexposed of the variables he believes to be con-

\section{KARGER \\ Fax +41613061234 E-Mail karger@karger.ch} www.karger.com
(C) 2011 S. Karger AG, Basel

$1660-2110 / 11 / 1184-0315 \$ 38.00 / 0$

Accessible online at:

www.karger.com/nec
Michiel A. de Graaf

Department of Clinical Epidemiology, Leiden University Medical Centre

Postzone C7-P, Postbus 9600

NL-2300 RC Leiden (The Netherlands)

E-Mail M.A.de_Graaf@LUMC.nl 


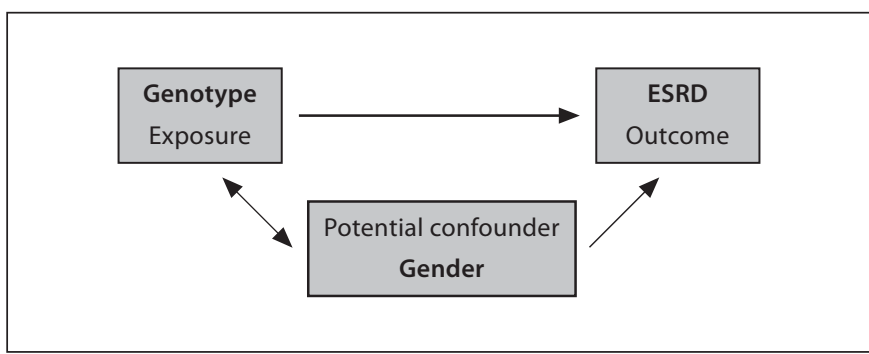

Fig. 1. Cases and controls matched on gender.

founding. This technique is referred to as matching. This can be done in two ways: individual matching when the researcher matches subject by subject, or frequency matching when the frequency of a variable is equally distributed among cases and controls [3]. Often, exposed and unexposed are matched for age and gender, but also more specific characteristics can be used, such as BMI, frequency of diabetes or blood pressure.

\section{Matching in a Cohort Study}

In a matched cohort study the exposed person is matched with one or more unexposed individuals (controls) on one or more confounders. A good example of a matched cohort study was presented by Ludvigsonn et al. [4] who investigated the relation between celiac disease (CD) and risk of renal disease. In this general population cohort study the authors used 14,336 patients with CD and 69,875 patients without CD matched for age, calendar year, sex, and county. As a result of this matching these variables had an equal distribution among both groups, therefore these variables had no effect as confounder. Based on this cohort, the investigators could easily calculate hazard ratios for glomerulonephritis and for renal replacement therapy, without these effect estimates being influenced by the confounding effect of age or sex. As a result they could demonstrate that individuals with CD had a higher risk of these events than others in the general population.

In contrast to matching in a case-control study (as explained further), in a matched cohort study further statistical analysis is not necessary. That only applies if no loss to follow-up or competing risk occur during followup. Logically if during follow-up individuals are lost, only the starting situation is matched. A disbalance in persons and person time at the end of the study can still occur. A difficulty of matching in a cohort study is the effort and costs it may require to collect matched controls, especially since cohort studies often contain large sample sizes.

\section{Matching in a Case-Control Study}

In a case-control study the starting point is a disease - where those having the disease are the cases and those without the disease are controls - and one looks back in time to study exposure. This study design provides an efficient way to investigate risk factors for a disease. It therefore is a suitable method for investigating rare diseases. In a matched case-control study, a case, affected by the disease, is matched with one or more individuals not affected by the disease, the controls. For example, the relation between hypertension and ESRD as described in the previous example could also be assessed in a case-control study. Cases would be patients with ESRD and controls the patients without ESRD. Based on the frequency of hypertension in both groups the odds ratio (OR) for hypertension on ESRD can then be calculated. To avoid confounding by BMI, researchers could match on BMI by ensuring an equal distribution of BMI among the patients with and without ESRD. Effectively, whereas in a cohort study matching is done to make exposure groups comparable on the matching variable, in a case-control study matching is done to make outcome groups comparable on the matching variable. Below, we explore the effect of matching in a case-control study to see whether it indeed has the intended effect of removing confounding.

Matched case-control studies are often performed in genetic association studies. For example, Ranganath et al. [5] studied the role of non-HLA genetic variants in ESRD. Cases were patients with ESRD, those with an elevated serum creatinine or with a creatinine clearance $<15 \mathrm{ml} /$ $\min / 1.73 \mathrm{~m}^{2}$. Controls without this renal impairment were sampled from the same geographical areas as the cases. Because gender was expected to be a confounder, cases and controls were matched on gender (fig. 1).

The cases showed a high number of male patients (85\%). As the controls were matched on gender they also included $85 \%$ males. Therefore, gender should no longer have an effect as confounder. In this paper the authors compared the frequencies of a genotype in cases and controls and used this frequency to calculate an OR. In both groups, blood samples were taken for genotyping of cytokine and ICAM-1 gene polymorphisms. This paper compares the frequencies of a genotype in the case and 
control group, and uses this frequency to calculate an OR. Although this appears to be an appealing and easy way to correct for a confounder, it might be incorrect. The following will clarify this further.

Another example of matched case-control is found in a study on smoking as a risk factor for primary renal diseases [6]. In this retrospective multicenter case-control study the smoking habits of patients with and without renal disease were analyzed. Patients were matched with controls on different variables, here we focus on gender. Of both cases and controls, smoking history was assessed using a standardized questionnaire. In their paper the result of these data are shown in a $2 \times 2$ table based on which an OR of 3.7 can be calculated. The univariate effect was of course the same as derived from the matched $2 \times 2$ table $(\mathrm{OR}=3.7)$. However, additional adjusting for the matching variable (gender) did increase the effect $(\mathrm{OR}=5.8)$. That is strange, because if matching would be sufficient to avoid the effect of gender, adjusting for gender using logistic regression should have no effect and the adjusted OR should equal the univariate, unadjusted OR of 3.7. So, the question remains - what is the correct OR?

The previous example shows that matching in a casecontrol study has an effect on the effect estimate that might be incorrect and unwanted. The explanation for this result can be demonstrated in the genetic association study by Ranganath et al. [5]. The researchers matched for a high frequency of male patients. However, by doing this the investigators introduced bias instead of avoiding a confounder. If the assumption that gender is a confounder is correct, that means that males have a higher risk of acquiring ESRD. Matching for this variable increases the risk of ESRD in the control group as, because of matching, the control group has a relatively high number of males and therefore a higher risk of ESRD than the general population. The relative effect of a genotype, calculated by the OR, is now lower than it would have been if compared to the general population. This means that in this study, matching has introduced bias by lowering the effect estimate of the several genotypes in the control group.

These examples show the problem that occurs when one makes use of matching in a case-control study. As an effect of matching on a confounder, case and control group become too similar not only in the distribution of the confounder (gender in both examples) but also in the distribution of the exposure (genotype and smoking, respectively). In other words, the cases and controls start to resemble each other on frequency of exposure. This results in a lower effect estimate, i.e. an OR closer to 1 . This can also be explained by a theoretical example. Suppose we would be able to match for all the variables affecting outcome. Both cases and controls would become almost completely similar resulting in an OR of approximately 1.

\section{Solution}

The previous paragraphs have shown that matching in a case-control study lowers the effect estimate. There are ways to correct for this lowered outcome in the analysis of the data. A Mantel-Haenszel OR or (conditional) logistic regression corrects for this disturbed frequency of the exposure. Mantel-Haenszel uses a weighing factor per stratum to correct for the size of a stratum, thereby taking in consideration the uneven distribution of a variable between cases and controls. The same can be achieved with (conditional) logistical regression. This is shown by the example of smoking as a risk factor for renal disease. Logistic regression gives a much higher $\mathrm{OR}$ than the one found by regular calculation. The above shows that in a case-control study, matching can only be used if the correct statistical method is used for removing the introduced bias in the matched control group.

\section{Individual Matching}

All study examples used frequency matching, meaning that the frequency of a confounder is equal in both groups. Matching is not done per individual subject but the overall distribution of a variable is made equal. Another way to match is subject matching; for each individual subject affected by a confounder, researchers search for an individual control affected by the confounder. In an individual matched case-control study ORs can be calculated using a matched-pair analysis [3]. For each matched pair you assess whether it gives information in line with or against the hypothesis, assuming the hypothesis that an association between risk factor and outcome is present. If the case has the risk factor and the matched control has not a pair is considered to be in line with the hypothesis. Vice versa, if the control has the risk factor but the case has not, this matched pair is considered to provide information against the hypothesis. There are ties if either both the case and the control have the risk factor or if the risk factor is absent in both case and control. Then the matched-pairs OR is calculated as the ratio of the number of pairs supporting the hypothesis over the number of pairs against the hypothesis, thereby ignoring 
all ties. If the number of pairs supporting the hypothesis is equal to the ones against it, the matched-pairs OR is 1 . In that case there is no effect seen of the risk factor on the outcome. However, if this OR is not equal to 1 , an effect is present.

\section{Why Matching?}

In this paper we discussed the difficulties that occur in a matched case-control study. Although matching in this study design does not solve the problems of confounding, it is still a commonly used technique. For confounders that are difficult to measure it could be a convenient way to sample controls to ask cases to bring a friend or relative as a control, which effectively implies individual matching. For example, the confounding effect of playing tennis is hard to measure, therefore a friend who also plays tennis is a suitable control. Matching also provides an easy way to sample controls. For example, matching on socioeconomic status can easily be done by asking patients to bring a friend or colleague. However, patients without friends or those for whom a matched control cannot be found will be automatically excluded from the analysis, introducing in bias and resulting in both lower statistical and logistical efficiency.

\section{Conclusion}

Matching appears to be an appealing way to avoid confounding in the design of a study. In a cohort study this can be done without any adverse effects on the effect estimate. In a case-control study, however, matching results in a lower (closer to 1) effect because cases and controls become too similar on the frequency of exposure. Matching does not completely solve the problem of confounding, but might be useful in special circumstances to the sample controls. A matched case-control study still requires statistical adjustments such as Mantel-Haenszel or conditional logistic regression analysis.

\section{References}

1 Van Stralen KJ, Dekker FW, Zoccali C, Jager KJ: Confounding. Nephron Clin Pract 2010; 116:c143-c147.

-2 Hsu CY, Iribarren C, McCulloch CE, Darbinian J, Go AS: Risk factors for end-stage renal disease: 25 -year follow-up. Arch Intern Med 2009;169:342-350.

3 Rothman KJ, Greenland S: Modern Epidemiology, ed 3. Philadelphia, Lippincott Williams \& Wilkins, 2008.
4 Ludvigsson JF, Montgomery SM, Olen O, Ekbom A, Ludvigsson J, Fored M: Coeliac disease and risk of renal disease - a general population cohort study. Nephrol Dial Transplant 2006;21:1809-1815.

\footnotetext{
5 Ranganath P, Tripathi G, Sharma RK, Sankhwar SN, Agrawal S: Role of non-HLA genetic variants in end-stage renal disease. Tissue Antigens 2009;74:147-155.

6 Orth SR, Hallan SI: Smoking: a risk factor for progression of chronic kidney disease and for cardiovascular morbidity and mortality in renal patients - absence of evidence or evidence of absence? Clin J Am Soc Nephrol 2008;3:226-236.
} 